\title{
Energy internet technology: modeling, optimization and dispatch of integrated energy systems
}

\author{
(C) Higher Education Press and Springer-Verlag GmbH Germany, part of Springer Nature 2018
}

With the rapid development of the information technology and the application of renewable energy, the energy internet, which has been widely accepted as a means of integrating energy flow, information flow, and business flow, is prone to be the future form of energy system. However, it is a new system, never existed in human history. Therefore, from philosophical imagination to engineering realization, there are many scientific and technical problems, waiting to be solved. The purpose of this special issue is to demonstrate the recent research works on energy internet, as well as some proposals and suggestions to decision makers for strategic planning.

The Chinese government has promulgated a number of policies on combining the internet with the smart energy system in order to construct a green, low carbon, high efficient, high security energy system. More than 50 demonstration projects for energy internet are under construction in deferent provinces of China. In this special issue, Yan analyzed the opportunities and challenges posed to developing the energy internet in the Yangtze River Delta. He proposed the framework of energy internet in the Yangtze River Delta and gave specific suggestions for the development of the energy internet in the Yangtze River Delta.

Many countries in Europe, as well as in America, also pay special attention to development of energy internet. Schwarz introduced the situation of energy internet in Germany. Amanpour and his co-workers researched

Received Nov. 16, 2018; online Dec. 10, 2018

Shilie WENG $(\bowtie)$, Yiwu WENG

School of Mechanical Engineering, Shanghai Jiao Tong University, Shanghai 200240, China

E-mail: slweng@sjtu.edu.cn

Chenghong GU

Department of Electronic \& Electrical Engineering, University of Bath, Bath, BA2 7AY, UK the energy generation and consumption demands in Germany and discussed the role of integrated energy systems in support of sustainable solutions for future energy transitions. In order to provide a targeted review for other countries, they analyzed different technologies (e.g. electric or gas fueled vehicles) for potential solutions.

The micro-energy network, perhaps, will be the fundamental element of the energy internet, and the distributed power generation system will be the most important infrastructure for power generation, energy conversion, and energy storage. Therefore, it is very urgent to greatly develop the distributed power generation system, including advanced energy storage technologies. The micro-gasturbine and fuel cell distinguish themselves from many promising distributed power generation technologies due to their good performance and environmental and ecological friendliness. Due to the non-toxicity, high power density, easy storage, and renewability of ethanol as a fuel including, the direct ethanol fuel cells (DEFCs) may be expected as an indispensable part of future energy internet technologies. However, one bottleneck problem should be solved in the wide application of direct ethanol fuel cells, i.e., to develop highly active anode materials to efficiently oxidize ethanol. Shen and her colleagues designed two special structures of palladium-based electro-catalysts for ethanol oxidation in alkaline direct ethanol fuel cells, i.e. solid solution alloy and near surface alloy architectures. The influences of palladium, iridium and gold on the activity were systematically evaluated and examined. It was demonstrated that the electro-catalyst with a near surface alloy design had a much higher catalytic activity (50.4\% enhancement) and better stability compared to the referred $\mathrm{Pd} / \mathrm{C}$. In order to fully understand the future development and technical bottleneck of the energy system of PV and MGT, Lv and her group presented the current development of the multi-energy complementary system combined with 
solar PV and MGT to improve the renewable energy conversion efficiency and to lower the operation risk of the system. The research not only considered system integration design and optimization technologies, but also energy management and control technologies that could guarantee the safe operation of the system and feed the real-time operation information back to the system underlying models. Finally, the future development was predicted. This paper was beneficial for designers and users to select the most appropriate technology for reasonable operation of a micro-energy system.

The energy internet has to be a multi energy system, including the electric-power system, the thermal energy system, the natural gas system, and the renewable energy system. The unique feature of energy internet is the so called "multi energy synergy". In recent years, aiming at the efficient and clean use of energy, and enhancement of the synergy effect of multi energy flow, it is a hot topic to investigate the mathematical model establishment and simulation /optimization of multienergy system, based on energy internet. The reason for this is that the multi-energy system is a complicated system with nonlinear multivariable, multiple physical and chemical processes, and time dependent characteristics. Targeted researches on the mathematical model and simulation/optimization of the energy system can effectively predict and improve the system performance. In this issue, Ren and his research group established a multi-objective optimization model for the investment plan and operation management of a hybrid distributed energy system. The overall annual cost and emissions of $\mathrm{CO}_{2}$ equivalents were selected as the objective functions. The non-dominated sorting generic algorithm II (NSGA-II) was employed to solve the optimization model, and the best compromised Pareto solution was determined based on the fuzzy set theory. Finally, they gave an illustrative example based on a hotel building to verify the effectiveness of the optimization model and the solving algorithm. Huo and his coworkers researched the optimization method for the interconnected energy hub system with combined ground source heat pump and borehole thermal storage. The method proposed can provide a new and effective way for making full use of ground source heat energy. Ma and his co-workers established a gas-electricity joint operation model and proposed a system evaluation index based on the energy quality character, considering the grade difference of the energy loss of the subsystem. Finally, the optimal scheduling approach was applied to the integrated energy system composed of an IEEE 39 nodes power system and a 10 nodes natural gas system according to the typical load characteristics of commercial and residential users. The penetration and impact of renewable energy in power grid system is another important and rapid research direction, because the randomness and instability of renewable energy will threaten the security of the power system. In this special issue, $\mathbf{L i}$ and his group proposed a dynamic programming method in smoothing ramp events in wind farms based on the energy internet model of wind power, pumped storage power station, and gas power station. They transformed the optimization problem in the energy internet into a multi-stage dynamic programming problem, and used evaluation functions to evaluate pollutant emissions. The results showed that the dynamic programming method proposed was effective for smoothing the wind power and reducing ramp events in energy internet. To avoid a huge waste of renewable energy resources, Yan and his co-workers conducted a cost-benefit analysis and comparison of the three techniques: direct curtailment, the reinforcement of networks to expand transfer capacity, and the conversion of excessive renewable into hydrogen to address wind curtailment. Their result showed that converting excessive wind power into hydrogen to transport was an environmentally friendly and cost-effective way to address wind curtailment. Furthermore, Ling studied optimal dispatch of the multienergy system using the power-to-gas technology considering flexible load on user side. A concrete analysis was conducted and an optimal dispatch of the system was verified via four different scenarios. The results indicated that cooperative dispatch of multienergy system using P2G was the most economical. In this condition, environmental effects and safe operation of the system could be guaranteed. Finally, Zhang provided a comprehensive energy solution for households employing a CCHP system. They described the inputs, outputs, conversion, and consumption process of multiple energies in households by the mathematic model of a home energy hub. In the research process, the concept of thermal comfort and a robust optimization model for HEH were developed. Their results showed that the comprehensive energy solution could realize multiple kinds of energy supplies with minimized total energy cost. Faccio and his group showed state-of-theart optimization methods to design the configuration of hybrid renewable energy systems. They conducted a detailed analyzes of different classification drivers considering design factors such as optimization goals and utilized optimization methods. Their initial results indicated that of all optimization goals, load demand parameters including loss of power supply probability and loss of load probability, cost, configuration, and environmental emissions are the most frequent design variables.

More importantly, the penetration of renewable energy will also have a great impact on and pose challenges to the energy economic market. Li summarized the key limitations in the EU market designs and network access toward renewable integration. Her 
research showed that two approaches were under ways: to make the market fit for renewable, and to set a practical example of how a competitive economy could be built on a sustainable and affordable energy system. The results can be of important reference to developing countries for perfecting the renewable energy market.

The coming decades will be a critical period for the development of the global energy internet, which requires the active participation of scientists, engineers, as well as governments, international organizations, social groups, and energy companies, to strengthen theoretical research, technological breakthroughs, and international cooperation, to jointly promote the construction of the global energy internet. Although the research scope in this special issue is limited, we hope that it can broaden and motivate readers' vision. We hope that readers will find some useful knowledge about energy internet in terms of technology, strategy, policy support, and future development.

\section{Editor's bio-sketches:}

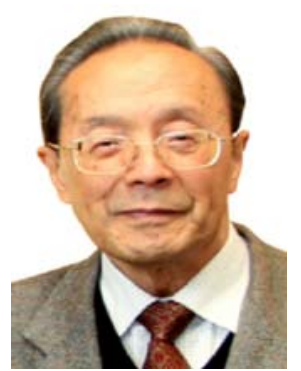

Dr. Shilie WENG, is a thermal engineering scientist mainly engaged in the research and development of thermal engines. He was elected as Academician of Chinese Academy of Engineering in 1995. He served as President of Shanghai Jiao Tong University from 1987 to 1997. Dr. WENG is one of the pioneers of the new generation of thermal turbines in China. He has worked in the research of multi-purpose modification of China's aero-turbine-fan engine and pioneered a new generation of China's thermal engine to improve the level of modernization. He developed the first prototype of China's ceramic adiabatic turbine composite diesel engine and completed the design and test bench construction of the first batch of turbocharger ceramic turbine rotors in China. As a chief scientist for the China high-tech program, he presided over the high-tech program of "Advanced wet air gas turbine cycle", "Basic construction project for marine gas turbine research" and etc. His work propels the development of thermal engines and modern gas turbine in China and around the world. E-mail: slweng@sjtu.edu.cn.

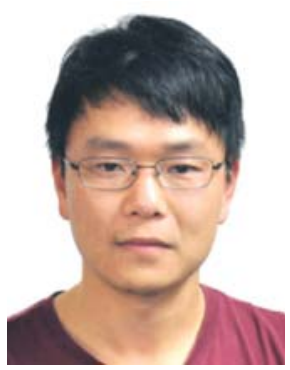

Dr. Chenghong GU is a Lecturer and EPSRC fellow in the Department of Electronic and Electrical Engineering, University of Bath, UK. His major research is in multi-vector energy system, smart grid and power economics. He obtained his Bachelor's and Master's degrees in electrical engineering in Shanghai Jiao Tong University in China in 2003 and 2007 respectively. In 2010, he obtained his Ph.D. in the University of Bath, UK. In the last five years, as the project leader, Dr GU's research has been supported by UK funding agency (EPSRC), the industry (NPG, NGC, and WPD), and the UK government (DECC). His research funds amounts to $£ 800$ thousand, and as the main contributor participates in the project about $£ 2$ million. He has published more than 60 papers, of which 40 have been retrieved by SCI journals, including IEEE Transactions on Power Systems and IEEE Transactions on Smart Grid. E-mail: cg277@bath.ac.uk.

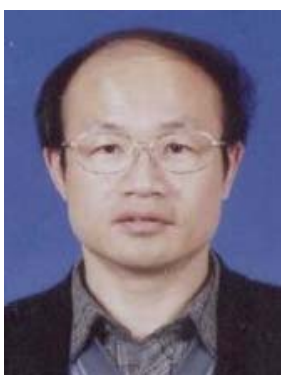

Dr. Yiwu WENG, is mainly engaged in research and development of energy and power systems. He serves as Professor and Deputy Dean of Energy Research Institute, Shanghai Jiao Tong University. He has won 3 provincial and ministerial Science and Technology Awards and has published more than 100 papers and 25 patents including 2 USA patents. He has involved in more than 20 national and provincial projects (including the 863 Project, the National Natural Science Foundation of China, the international cooperation projects and national defence military projects of the Ministry of Science and Technology, major projects of Shanghai Science and Technology Commission etc.), such as "The Low Calorific Gas Turbine Power System using Coal Mine Ventilation Gas", "The Development Program of Fuel Cell /Gas Turbine Hybrid Power System”. E-mail: ywweng@sjtu.edu.cn. 